\title{
How can the quality of life of older patients living with chronic pain be improved?
}

\author{
Wilco P Achterberg*,1 (iD) \\ ${ }^{1}$ Department of Public Health \& Primary Care, Leiden University Medical Center, Leiden, the Netherlands \\ *Author for correspondence: Tel.: +31 71526 8412; Fax: +31 62366 8980; w.p.achterberg@lumc.nl
}

\begin{abstract}
"More studies should be performed in the groups of older individuals and in the even more vulnerable groups such as people with dementia. Improved and promising observational assessment instruments based on earlier research have been developed and deserve to be further implemented and validated"
\end{abstract}

First draft submitted: 16 June 2019; Accepted for publication: 2 July 2019; Published online: 27 August 2019

Keywords: multimorbidity dementia $\bullet$ pain assessment $\bullet$ pain management $\bullet$ quality of life

\section{Epidemiology of pain in older individuals}

More and more people are reaching old age. Today, 125 million people are aged 80 years or older and by 2050, it is predicted that there will be 434 million people in this age group worldwide [1]. This aging process goes together with many more years of relative health and functional independency, but unfortunately eventually also comes with multimorbidity and impairments. There are many unwanted fellow travelers in this process such as chronic diseases, social isolation and functional impairments that seriously challenge quality of life (QoL). Chronic pain is also one of those unwanted guests that is hitting the oldest population the hardest with prevalence rates of $72 \%$ above the age of 85 years [2]. Not all types of pain are more frequent in old age: some types of pain, such as angina pectoris seem to decrease with age, but others such as neuropathic and musculoskeletal pain seem to reach a maximum at higher age [3]. The back and leg, knee and hip joints are the most common locations for pain in older individuals [4].

\section{Impact of pain in older individuals}

Pain in older individuals can have a negative influence on QoL in many ways. It can seriously hamper everyday functioning, is related to depression and is considered by the elderly themselves to be one of the biggest health inconveniences. This is true for older persons living in the community [5], but also for those who live in healthcare institutions.

In residents with dementia living in a nursing home, undertreated pain may trigger behavioral disturbances, mood syndromes, and deterioration of physical functioning and self maintenance [6]. And although pain is difficult to assess in persons with dementia, the impact on QoL is probably considerable. Also, it has been shown that pain can be a hindrance for the success of interventions that can help improve QoL: a psychosocial intervention with personalized care in persons with dementia showed that it did not work if the patients were in pain [7]. Also, a systematic review found that effective pain management can improve other outcomes, such as challenging behavior [8]. In addition, sleeping and night-time behaviors, social activities and mood and apathy, improved after adequate pain management - interestingly, this was irrespective of whether or not pain was present. Good pain management has also been proven to be beneficial for staff distress, activities of daily living, appetite and eating disturbances [9].

\section{Barriers to pain management in older individuals}

For many years it has been found in studies that pain in older patients is not managed well, and that the older you get, the worse it will be [3]. Many barriers regarding identification, assessment and management of pain in older people have been described in the literature. Barriers can be roughly distinguished in patient and provider factors that may contribute to the under treatment of pain [4]. 
Ageism

Ageism is a prejudice or discrimination on the grounds of a person's age. Healthcare workers, such as doctors, might believe that pain is normal in aging, and even older patients themselves might think so - this is known as self ageism.

Elderly individuals might be reserved in discussing their pain, because of stoicism in their attitude toward pain, because they do not want to be a complainer and because they have other, often functional, challenges in their everyday life which they give priority in their contacts with healthcare professionals.

\section{Communication/cognitive problems}

Pain identification may be substantially hindered due to communication problems in aphasia after stroke or in the case of dementia. As a result, people suffering from dementia receive less analgesics or reach later equivalent doses of analgesics compared with people without cognitive impairment [10]. Cognitive impairment can have a major impact on pain management, as the key factor of pain management is communication; knowing where the pain is, when it is present, how it influences everyday life, what ameliorates it, and knowing what management strategies are successful depend on the patient's ability to communicate effectively. In patients with dementia, these communications skills are often impaired, which makes recognition, management and evaluation of pain quite a challenge [10].

\section{Lack of evidence}

Another factor that influences pain management is the relative lack of studies on pain management in older individuals. Both pharmacological and nonpharmacological studies are usually performed in populations under the age of 65 , using individuals with relatively little comorbidity or polypharmacy, and with good cognitive skills. So, strong evidence for the efficacy is not often available. While there is clinical reason to be cautious of side effects, many organs such as kidneys and liver might work suboptimally in a frail body with multimorbidity, polypharmacy and cognitive impairment.

There are many reasons to believe that this situation seriously affects the QoL of older individuals and those with cognitive impairment. If we want the population to age happily, we have to take on the challenge of improving pain management for the elderly.

\section{Next steps}

Older individuals have the right to good recognition, assessment and management of their pain. Pain assessment in general should be conducted on a regular basis. In every setting the assessment of all aspects of pain is paramount, including emotional and functional aspects. Pain assessment should occur during the first interaction with the patient and follow-up will vary depending on the severity of pain, type of institution and needs of the patient.

In a hospital, regular pain assessment, preferably two to three times per day, should be conducted as long as sufficient pain relief has been achieved. A full assessment should be performed after an incident like a fall, or when the patient is not improving according to expectations.

In long-term care settings, attention for pain and its consequences should be part of everyday regular contacts. Therefore, pain assessments should be conducted whenever it is deemed to be necessary and helpful to the patient and until sufficient pain control is reached.

The consequences of pain are not only relevant for the assessment but may also help in the identification of adequate management strategies.

Physicians must exercise restraint in prescribing NSAIDs and weak opioids in vulnerable older persons, because of dangerous cardiovascular, renal and gastrointestinal side effects. Paracetamol is a relatively safe and is therefore good first choice medicine. For all therapies it is important to monitor and evaluate and terminate treatment in a timely manner if required. However, one should not be too conservative in prescribing opioids because of fear of addiction, as addiction is more likely in younger persons [11]. One should, however, realize that for opioid prescription the geriatric adage 'Start Low, Go Slow' is very important, and 'Stop in Time' is a very important addition to that rule [12].

Nonpharmacological strategies are helpful in reducing the negative impact of pain on QoL. These include relaxation therapies, cognitive strategies, transcutaneous electrical nerve stimulation in addition to physiotherapy and occupational therapy to help the patient overcome the functional impairments that often are related to chronic pain in older persons [4]. 


\section{Future perspective}

First, we must face a societal challenge: we have to fight ageism. In the coming decades, with so many people living to older ages, society cannot maintain the idea that pain in older persons is normal. There should be action to educate both medical professionals and the general public about these issues. Most importantly, the elderly individuals themselves must be aware that pain is not an unavoidable factor of old age. Second, an educational agenda must be established. Healthcare workers need to be informed about aging and the complicated relationship between aging and pain - both the biological, psychological and social aspects. This will allow healthcare workers to be better prepared for multimorbidity, frailty and other more complex problems associated with old age. Important educational issues also refer to assessment, and the challenges of assessment in older persons. Next to the well-known and regularly used instruments such as the Visual Analogue Scale and Numerical Rating Scale, other assessment instruments such as Faces Pain Scale and observational pain scales should be in the repertoire of persons caring for older persons.

Third, we have a research agenda. More studies should be performed in the groups of older individuals and in the even more vulnerable groups such as people with dementia. Improved and promising observational assessment instruments based on earlier research have been developed and deserve to be further implemented and validated [13,14]. Pharmacologically, it is important to study dose-effect relations, drug-drug interactions as well as drug-disease interaction in frail subjects. Nonpharmacological interventions including additional interventions that are focused on the consequences of pain, such as functional impairment should also be further developed. Special attention, both in research and education, should be placed on the challenges of pain assessment and management in the growing vulnerable elderly population.

\section{Financial \& competing interests disclosure}

The author has no relevant affiliations or financial involvement with any organization or entity with a financial interest in or financial conflict with the subject matter or materials discussed in the manuscript. This includes employment, consultancies, honoraria, stock ownership or options, expert testimony, grants or patents received or pending, or royalties.

No writing assistance was utilized in the production of this manuscript.

\section{References}

1. WHO. Ageing and health (2019). www.who.int/news-room/fact-sheets/detail/ageing-and-health

2. Duncan R, Francis RM, Collerton J et al. Prevalence of arthritis and joint pain in the oldest old: findings from the Newcastle $85+$ study. Age Ageing 40(6), 752-755 (2011).

3. Savvas SM, Gibson SJ. Overview of pain management in older adults. Clin. Geriatr. Med. 32(4), 635-650 (2016).

4. Abdulla A, Adams N, Bone M et al. Guidance on the management of pain in older people. Age Ageing 42(Suppl. 1), i1-i57 (2013).

5. van Blijswijk SC, Chan OY, van Houwelingen AH, Gussekloo J, den Elzen WP, Blom JW. Self-reported hindering health complaints of community-dwelling older persons: a cross-sectional study. PLoS ONE 1610(11), e0142416 (2015).

6. van Dam PH, Caljouw MAA, Slettebø DD, Achterberg WP, Husebo BS. Quality of life and pain medication use in persons with advanced dementia living in long-term care facilities. J. Am. Med. Dir. Assoc. doi:10.1016/j.jamda.2019.02.019 (2019) (Epub ahead of print).

7. Stacpoole M, Hockley J, Thompsell A, Simard J, Volicer L. The Namaste Care programme can reduce behavioural symptoms in care home residents with advanced dementia. Int. J. Geriatr. Psychiatry 30(7), 702-709 (2015).

8. Pieper MJ, van Dalen-Kok AH, Francke AL et al. Interventions targeting pain or behaviour in dementia: a systematic review. Ageing Res. Rev. 12(4), 1042-1055 (2013).

9. Husebo BS, Ballard C, Fritze F, Sandvik RK, Aarsland D. Efficacy of pain treatment on mood syndrome in patients with dementia: a randomized clinical trial. Int. J. Geriatr. Psychiatry 29(8), 828-836 (2014).

10. Achterberg WP, Pieper MJ, van Dalen-Kok AH et al. Pain management in patients with dementia. Clin. Interv. Aging 8, 1471-1482 (2013).

11. Webster LR. Risk factors for opioid-use disorder and overdose. Anesth. Analg. 125(5), 1741-1748 (2017).

12. Achterberg W. Pain management in long-term care: are we finally on the right track? Age Ageing 45(1), 7-8 (2016).

13. Corbett A, Achterberg W, Husebo B et al. An international road map to improve pain assessment in people with impaired cognition: the development of the Pain Assessment in Impaired Cognition (PAIC) meta-tool. BMC Neurol. 10(14), 229 (2014).

14. Ersek M, Neradilek MB, Herr K et al. Psychometric evaluation of a pain intensity measure for persons with dementia. Pain Med. 20(6), 1093-1104 (2018). 
